\title{
Charge distribution effects in the solution chemistry of polyatomic ions
}

\author{
W.R.Fawcett \\ Department of Chemistry University of California Davis, CA 95616
}

Received November 15, 2004

The distribution of charge within polyatomic ions determined in ab initio quantum chemical calculations is used to describe the solution chemistry of these species in more detail. Two examples are considered in this paper. The solvation of small polyatomic ions such as nitrate and perchlorate is considered with respect to the MSA model for ion solvation. The second example involves a consideration of double layer effects for transition metal complexes undergoing electron transfer in the double layer.

Key words: solvation, polyatomic ions

PACS: 82.60.Lf

Presented at the Symposium honoring D.Henderson on the occasion of his 70th birthday which took place at Brigham Young University on August 7, 2004.

\section{Introduction}

It is common in the development of statistical thermodynamic models of electrolyte solutions to represent the ions of the electrolyte as point charges embedded in hard spheres of a given radius. Such a representation is probably reasonable in the case of monoatomic ions. Thus, $\mathrm{NaCl}$ can be represented as two hard spheres, one for the $\mathrm{Na}^{+}$cation with its corresponding radius, and another for the $\mathrm{Cl}^{-}$anion with its radius. In a non-primitive description of an aqueous solution of $\mathrm{NaCl}$, the solvent can be modeled as a third sphere with an embedded dipole. In simpler, primitive models the solvent is represented as a dielectric continuum. Examination of the literature [1] involved with the statistical thermodynamic description of water shows that the point-dipole-hard sphere model for water is inadequate. In fact, Guillot has listed 46 more detailed models for water beginning with the seminal work of Bernal and Fowler [2]. One successful way of improving the description of water is to determine the charge distribution in an individual water molecule using 
$a b$ initio quantum mechanics, and then use this information in a site-site solution of the Ornstein-Zernike equation [3].

It is also not surprising that the point charge- hard sphere model is inadequate in the description of the solution properties of polyatomic ions. In order to understand the properties of these systems one must have a detailed knowledge of the charge distribution within the ion so that one can explain how it interacts with the surrounding medium. In this paper two aspects of this problem are considered. The first is the solvation of polyatomic ions in polar solvents. The second aspect is the double layer effect observed for redox couples undergoing electron transfer in the double layer near a polarizable electrode.

Table 1. Parameters for ion solvation.

\begin{tabular}{|c|c|c|}
\hline \multicolumn{3}{|c|}{ Monoatomic Ions } \\
\hline $\mathrm{Ion}$ & $r, \mathrm{pm}$ & $\Delta_{\mathrm{s}} G, \mathrm{~kJ} \mathrm{~mol}$ \\
$\mathrm{Li}^{+}$ & 88 & -529 \\
$\mathrm{Na}^{+}$ & 116 & -424 \\
$\mathrm{~K}^{+}$ & 152 & -352 \\
$\mathrm{Rb}^{+}$ & 163 & -329 \\
$\mathrm{Cs}^{+}$ & 184 & -306 \\
$\mathrm{Mg}^{2+}$ & 86 & -1931 \\
$\mathrm{Ca}^{2+}$ & 114 & -1608 \\
$\mathrm{Sr}^{2+}$ & 130 & -1479 \\
$\mathrm{Ba}^{2+}$ & 150 & -1352 \\
$\mathrm{~F}^{-}$ & 119 & -429 \\
$\mathrm{Cl}^{-}$ & 167 & -304 \\
$\mathrm{Br}^{-}$ & 182 & -278 \\
$\mathrm{I}^{-}$ & 206 & -243 \\
$\mathrm{~S}^{2-}$ & 170 & -1238 \\
\hline \multicolumn{3}{|c|}{ Polyatomic ions } \\
\hline $\mathrm{NH}_{4}^{+}$ & $153^{*}$ & -340 \\
$\mathrm{NO}_{3}^{-}$ & $198^{*}$ & -258 \\
$\mathrm{ClO}_{4}^{-}$ & $309^{*}$ & -165 \\
\hline
\end{tabular}

* Estimated from the linear plots in figure 1 (see text).

\section{The solvation of polyatomic ions}

In the case of monoatomic ions, the thermodynamic property which has attracted the most attention is the Gibbs energy of solvation $\Delta_{\mathrm{S}} \mathrm{G}$ [4]. This quantity is obtained from thermodynamic data estimated for electrolyte solutions in the limit of infinite dilution, and requires an extra-thermodynamic assumption so that the contributions of the cation and anion can be separated. A very effective way of obtaining the single ion quantities is measuring the Gibbs energy of formation of 
ion-water clusters in the gas phase using mass spectrometry [5]. Values of $\Delta_{\mathrm{S}} \mathrm{G}$ for common monatomic cations and anions are summarized in table 1 . These data were obtained from the thermodynamic results for electrolytes assuming that the value of $\Delta_{\mathrm{s}} \mathrm{G}$ for the proton, $\mathrm{H}^{+}$is $-1104 \mathrm{~kJ} \mathrm{~mol}^{-1}$ [5]. Also recorded are the Shannon and Prewitt radii for these ions [6]. These radii may be regarded as improved Pauling radii which were extracted from X-ray crystallographic data for a large collection of ionic crystals [6].

The simplest description of ionic solvation is that based on the Born model [4]. The ion is assumed to be a charged hard sphere in a dielectric continuum corresponding to the solvent. The Gibbs energy of salvation is estimated on the basis of the work done to discharge the ion in vacuo, and then recharge within the dielectric medium. The model leads to the following expression for $\Delta_{\mathrm{S}} G_{i}$ for ion i with radius $r_{i}$ :

$$
\Delta_{\mathrm{s}} G_{i}=\frac{-N_{L} z_{i}^{2} e_{0}^{2}}{8 \pi \varepsilon_{0} r_{i}}\left(1-\frac{1}{\varepsilon_{\mathrm{s}}}\right)
$$

Here, $N_{L}$ is the Avogadro constant, $z_{i}$, the charge number of the ion, $e_{0}$, the fundamental electronic charge, $\varepsilon_{0}$, the permittivity of free space, and $\varepsilon_{\mathrm{s}}$, the relative permittivity of the solvent. This equation suggests that $\Delta_{\mathrm{S}} \mathrm{G}_{i}$ should be proportional to $z_{i}^{2} / r_{i}$. However, this is clearly not the case as can be seen from the data for monatomic ions in table 1 . The Born model fails because it does not recognize the disruption of solvent structure which occurs in the immediate vicinity of the ion. In addition, it does not deal with the fact that the molecular mechanism involved in cation solvation is different from that for anions. In water cations are solvated by the electron density on the oxygen atom, whereas anions are solvated by hydrogen bonding with the water molecules.

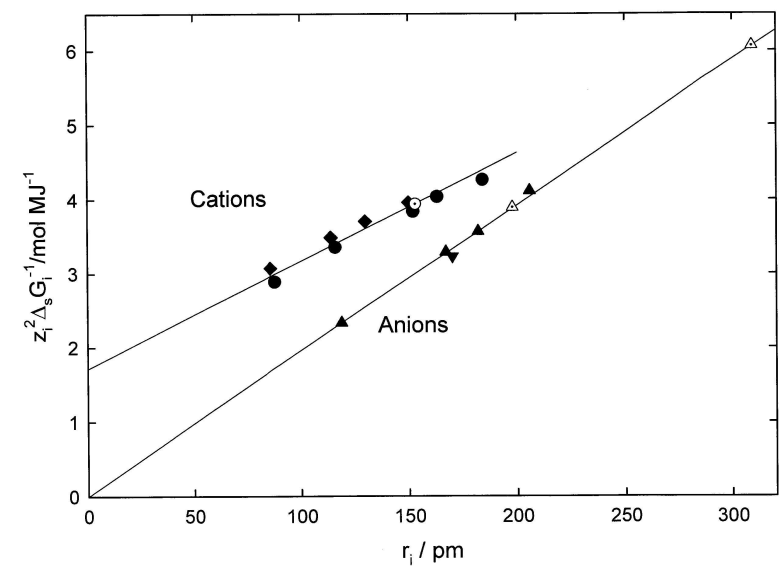

Figure 1. Plot of $z_{i}^{2} \Delta_{\mathrm{s}} G_{i}^{-1}$ against the ionic radius $r_{i}$ for the alkali metal $(\bullet)$ and alkaline earth metal cations $(\boldsymbol{\vee})$, and for the halide $(\boldsymbol{\Delta})$ and sulfide $(\boldsymbol{\nabla})$ anions. The cationic data have been shifted vertically by $1 \mathrm{~mol} \mathrm{MJ}^{-1}$ for the sake of clarity. The data for $\mathrm{NH}_{4}^{+}(\circ), \mathrm{NO}_{3}^{-}$and $\mathrm{ClO}_{4}^{-}(\triangle)$ are placed on the lines according to their values of $z_{i}^{2} \Delta_{\mathrm{s}} G_{i}^{-1}$. 
A better description of ion solvation is provided by the mean spherical approximation (MSA) used to obtain a solution of the Ornstein-Zernike integral equation describing ion-solvent interactions in the electrolyte solution [4]. The approach involved is non-primitive, the ions being represented as charged hard spheres, and the solvent molecules as hard spheres with embedded point dipoles. The MSA model gives the following expression for the Gibbs energy of solvation:

$$
\Delta_{\mathrm{s}} G_{i}=\frac{-N_{L} z_{i}^{2} e_{0}^{2}}{8 \pi \varepsilon_{0}}\left(\frac{1}{r_{i}+\delta_{\mathrm{s}}}\right)\left(1-\frac{1}{\varepsilon_{\mathrm{s}}}\right) .
$$

Equation (2) differs from the Born expression by the term in $\delta_{\mathrm{s}}$ which corrects the ion radius by an amount which depends on the nature of the solvent. In fact, $\delta_{\mathrm{s}}$ is equal to the solvent radius divided by the Wertheim polarization parameter $\lambda_{\mathrm{s}}$. In the case of water, $\delta_{\mathrm{s}}$ is equal to $54 \mathrm{pm}$. The MSA equation suggests that one should plot $z_{i}^{2} / \Delta_{\mathrm{s}} G_{i}$ against $r_{i}$ to test the model. Separate plots for the monoatomic cations and anions listed in table 1 are shown in figure 1. Linear plots are obtained for the two sets of data but they have different slopes and intercepts. This analysis points out the differences in the way cations and anions interact with the solvent molecules.

The best straight line through the cationic data is

$$
z_{i}^{2} / \Delta_{\mathrm{S}} G_{i}=0.71+0.0146 r_{i} .
$$

The value of $N_{L} e_{0}^{2} / 8 \pi \varepsilon_{0}$ is $69.45 \mathrm{MJ} \mathrm{pm}^{-1}$. When the factor $\left(1-1 / \varepsilon_{\mathrm{s}}\right)$ is included the proportionality constant in equation (2) becomes $68.57 \mathrm{MJ} \mathrm{pm}^{-1}$. Thus, the slope of equation (3) is very close to that predicted by equation (2). From the intercept, the estimate of $\delta_{\mathrm{s}}$ is $49 \mathrm{pm}$. This is smaller than the value predicted theoretically (54 pm).

Cation<smiles>OCO</smiles>

(a)
Anion

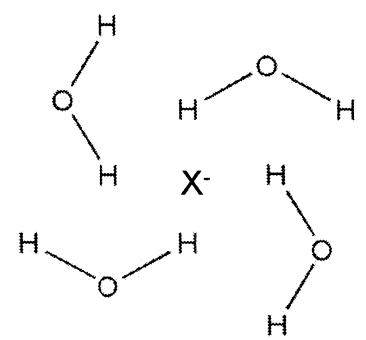

(b)

Figure 2. Structure of water molecules in the primary solvation layer around a cation (a) and an anion (b).

Analysis of the data for anions leads to quite a different result:

$$
z_{i}^{2} / \Delta_{\mathrm{S}} G_{i}=0.0196 r_{i} .
$$


Not only is the parameter $\delta_{s}$ equal to zero but the slope of the plot is considerably higher than predicted by equation (2). On the basis of the MSA model [4], this can be attributed to the failure of the model to account for repulsive dipole-dipole interactions between the dipoles immediately surrounding the ion. An approximate way of including this effect in the MSA result is to write equation (2) as

$$
\Delta_{\mathrm{s}} G_{i}=\frac{N_{L} z_{i}^{2} e_{0}^{2} f_{\mathrm{dd}}}{8 \pi \varepsilon_{0}}\left(\frac{1}{r_{i}+\delta_{\mathrm{s}}}\right)\left(1-\frac{1}{\varepsilon_{\mathrm{s}}}\right),
$$

where the fraction $f_{\text {dd }}$ is less than unity when repulsive dipole-dipole interactions are important. For the monovalent anions $f_{\text {dd }}$ is equal to 0.76 .

The proposed structure of water around cations and anions is illustrated in figure 2. In the case of cations which are solvated by the electron density on the oxygen atom of the water molecule, water structure around the cation is significantly disrupted so that $\delta_{s}$ is large. However, the orientation of the solvating dipoles with respect to one another is such that repulsive dipole-diploe interactions are not important. In the case of anions which are solvated by hydrogen bonding (see figure 2), the local water structure is much less disrupted, one hydrogen bond on each solvating water molecule being free to interact with water molecules further from the anion. The mutual orientation of the solvating water dipoles is such that repulsive dipole-dipole interactions are important.

Table 2. Geometry and charge distribution in two polyatomic anions.

\begin{tabular}{|l|l|l|}
\hline Anion & Nitrate & Perchlorate \\
\hline Geometry & Trigonal (planar) & Tetrahedral \\
Charge on central atom & $0.86 e_{o}($ on N) & $1.88 e_{o}($ on $\mathrm{Cl})$ \\
Charge on outer oxygens & $-0.62 e_{o}$ & $-0.72 e_{o}$ \\
Bond length & $127 \mathrm{pm}(\mathrm{N}-\mathrm{O})$ & $150 \mathrm{pm}(\mathrm{Cl}-\mathrm{O})$ \\
Bond angle & $120(\mathrm{O}-\mathrm{N}-\mathrm{O})$ & $109(\mathrm{O}-\mathrm{Cl}-\mathrm{O})$ \\
\hline
\end{tabular}

The linear plots shown in figure 1 may be used to define effective radii for the polyatomic ions which are also listed in table 1 . In order to understand these results and the solvation of polyatomic ions one needs to know the charge distribution within the ion. Krienke and Schmeer recently carried out ab initio calculations for the nitrate and perchlorate ions [3]. The nitrate ion is planar with angles of $120^{\circ}$ between the three $\mathrm{N}-\mathrm{O}$ bonds. In this system the charge on each oxygen atom is $-0.62 e_{o}$ whereas the charge on the central nitrogen atom is $0.86 e_{o}$ (see table 2). Since all four atoms can be reached by the surrounding water molecules, the nitrate ion is strongly solvated. As a result, the solubility of nitrate salts in water is very high. On the other hand, nitrate salts do not dissolve significantly in polar aprotic solvents. These solvents are typically strong Lewis bases and weak Lewis acids. Thus, solvation of the positive nitrogen atom would dominate in the non-aqueous medium. Due to the larger size of the organic solvent molecule, solvation of the 
negative oxygen atoms is probably prevented by steric effects. Finally, it is noted that the effective radius of nitrate $(198 \mathrm{pm})$ is larger than the length of the $\mathrm{N}-\mathrm{O}$ bond $(122 \mathrm{pm})$. Thus, the effective radius estimated on the basis of the MSA model is reasonable within the context of the structural information for this anion [3].

Perchlorate anion has a tetrahedral structure with a large positive charge on the central chlorine atom $\left(1.88 e_{o}\right)$ and a small negative charge on each of the oxygen atoms $\left(-0.72 e_{o}\right)$. Since the chlorine atom is isolated from the surrounding solvent, it is not involved in the solvation process. Due to its tetrahedral structure, perchlorate fits easily into the water structure in a non-disruptive manner. Each of the oxygen atoms are hydrogen bonded to adjacent water molecules. This accounts for the high solubility of $\mathrm{LiClO}_{4}$ and $\mathrm{NaClO}_{4}$ in water. These electrolytes have strongly solvated cations. However, $\mathrm{KClO}_{4}$ is only sparingly soluble in water, presumeably because the $\mathrm{K}^{+}$cation can interact directly with one of the oxygen atoms of the perchlorate anion. Perchlorate electrolytes are also known to be easily dissolved in polar aprotic solvents [7]. In these systems the negative charge on each oxygen atom is solvated by the positive end of the molecular dipole in the solvent molecule. Due to its tetrahedral geometry, steric effects in solvation of this anion are not a problem. Finally the effective radius of the perchlorate ion $(309 \mathrm{pm})$ is larger than the length of the $\mathrm{Cl}-\mathrm{O}$ bond $(150 \mathrm{pm})$. The usefulness of effective radii defined on the basis of the MSA model remains to be tested for other thermodynamic properties. However, the results obtained in the present study appear reasonable within the context of the quantum chemical calculations [3].

Table 3. Geometry and charge distribution in two polyatomic cations.

\begin{tabular}{|l|l|l|}
\hline Cation & {$\left[\mathrm{Co}\left(\mathrm{NH}_{3}\right)_{6}\right]^{3+}$} & {$\left[\mathrm{Cr}\left(\mathrm{H}_{2} \mathrm{O}\right)_{6}\right]^{3+}$} \\
\hline Geometry & Octahedral & Octahedral \\
Charge on central atom & $1.67 e_{o}($ on $\mathrm{Co})$ & $2.12 e_{o} e_{o}($ on $\mathrm{Cr})$. \\
Metal-Ligand distance & $205 \mathrm{pm}(\mathrm{Co}-\mathrm{N})$ & $202 \mathrm{pm}(\mathrm{Cr}-\mathrm{O})$ \\
Charge on central ligand atom & $-1.19 e_{o}($ on $\mathrm{N})$ & $-1.02 e_{o}(\mathrm{on} \mathrm{O})$ \\
Bond length in ligand & $101 \mathrm{pm}(\mathrm{N}-\mathrm{H})$ & $96 \mathrm{pm}(\mathrm{O}-\mathrm{H})$ \\
Charge on ligand hydrogens & $0.47 e_{o}$ & $0.58 e_{o}$ \\
\hline
\end{tabular}

\section{Double layer effects}

In the Frumkin model for double layer effects in heterogeneous electron transfer kinetics [8], the reactant is assumed to be a point charge located on the outer Helmholtz plane (oHp). However, in reality most reactants which undergo simple electron transfer are polyatomic ions whose dimensions are comparable with the thickness of the diffuse layer. Two examples of systems used to study simple electron transfer kinetics are $\left[\mathrm{Co}\left(\mathrm{NH}_{3}\right)_{6}\right]^{3+/ 2+}$ and $\left[\mathrm{Cr}\left(\mathrm{H}_{2} \mathrm{O}\right)_{6}\right]^{3+/ 2+}$. From the charge and 


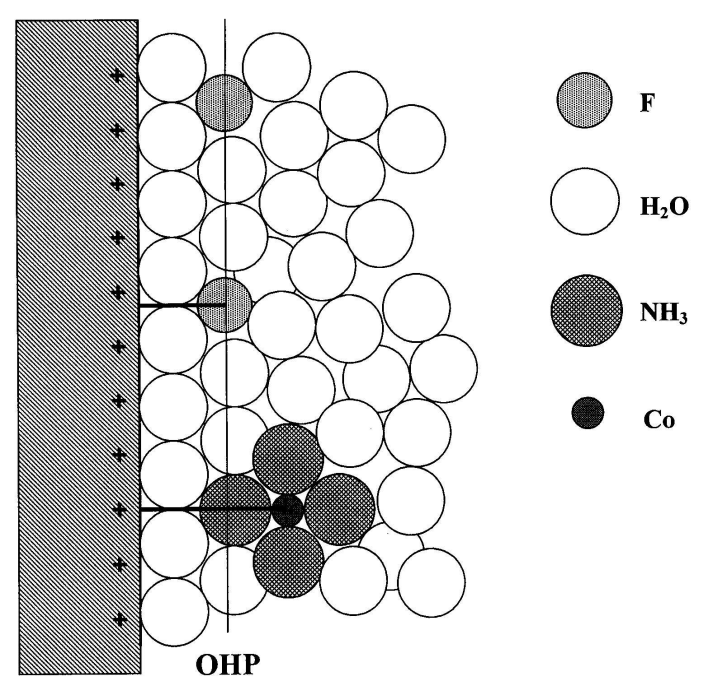

Figure 3. Composition of the double layer near the oHp at a positively charged electrode with $\mathrm{NaF}$ as electrolyte and $\left[\mathrm{Co}\left(\mathrm{NH}_{3}\right)_{6}\right]^{3+}$ as reactant.

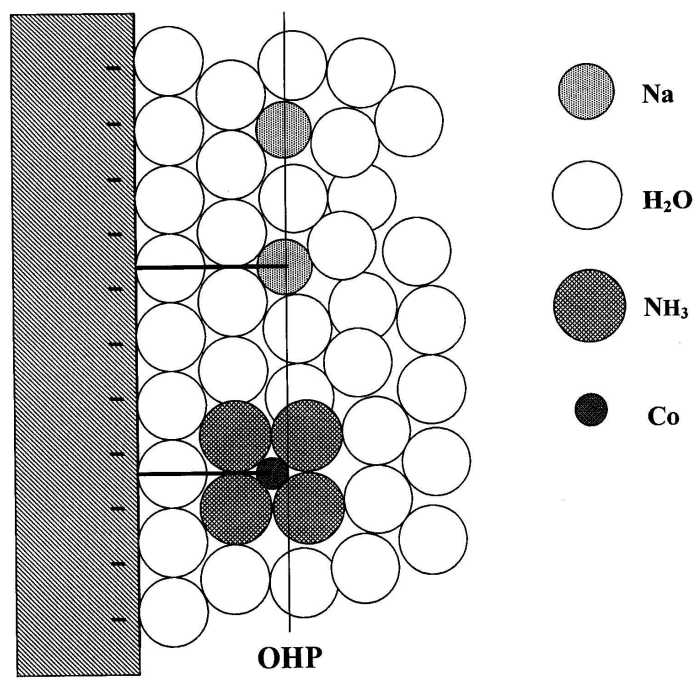

Figure 4. Composition of the double layer near the oHp at a negatively charged electrode with $\mathrm{NaF}$ as electrolyte and $\left[\mathrm{Co}\left(\mathrm{NH}_{3}\right)_{6}\right]^{3+}$ as reactant.

bond length information obtained in ab initio quantum chemical calculations $[9,10]$, these ions have effective radii greater than $300 \mathrm{pm}$ (see table 3 ).

This is significant with respect to the Debye length in a $0.1 \mathrm{M} 1-1$ electrolyte $(\approx 964 \mathrm{pm})$. It follows that a more careful consideration of the double layer effect requires that one should consider the variation in potential with the position in the reactant. Therefore, one must assume both a position and an orientation for the reactant in the double layer. Typical situations for an octahedral complex such as $\left[\mathrm{Co}\left(\mathrm{NH}_{3}\right)_{6}\right]^{3+}$ are illustrated in figures 3 and 4 . When a positively charged reactant undergoes electron transfer at a positively charged electrode, it is oriented at its distance of closest approach so that the minimum amount of positive charge is closest to the electrode. This means that one ligand is close to the electrode, four are in axial positions, and the sixth is at the farthest distance from the electrode (figure 3). Given the description of the average potential as a function of distance from the oHp, one may estimate an effective charge $z_{\text {eff }}$ for the reactant which is defined as

$$
z_{\mathrm{eff}}=\frac{\sum_{i} z_{i} \phi^{i}}{\phi^{d}}
$$

where $z_{i}$ is the charge on atom $i$ of the reactant, $\phi^{i}$, the potential at this atom, and $\phi^{d}$, the potential at the oHp. In the example chosen, the position of the oHp is determined by the unsolvated fluoride anion which is the predominant counter ion at positive potentials for this system. Furthermore, most of the atoms in the complex ion are further from the oHp in the diffuse layer where the local potential is smaller in magnitude. As a result, the effective charge for the $\left[\mathrm{Co}\left(\mathrm{NH}_{3}\right)_{6}\right]^{3+}$ reactant is expected to be significantly less than +3 . 
The probable position and orientation of the same reactant at a negatively charged interface is shown in figure 4 . In this case, the position of the oHp is assumed to be determined by a solvated $\mathrm{Na}^{+}$cation, and is therefore further from the metal/ solution interface than when the surface is positively charged. Furthermore, the orientation of the reactant is such that the maximum number of positively charged $\mathrm{NH}_{3}$ ligands is closer to the electrode. As a result these ligands are in a region of the double layer where the electrical field is high. It follows that the double layer effect is expected to be more important, and the effective charge on the reactant larger under these circumstances. Of course, the details shown in figures 3 and 4 are necessarily speculative, but they are based on reasonable assumptions and include realistic sizes for the ionic and molecular components when they are represented as hard spheres.

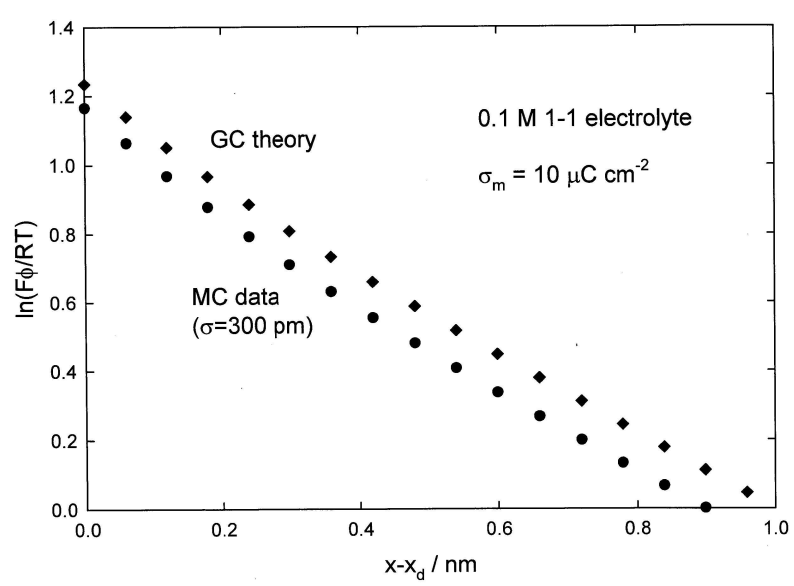

Figure 5. Potential profiles in the diffuse layer plotted as $\ln \left(F_{\phi} / R T\right)$ against position in the diffuse layer, $x-x_{d}$. The data $(\downarrow)$ were calculated using GC theory for an electrolyte concentration of $0.1 \mathrm{M}$ and an electrode charge density of $10 \mathrm{C} \mathrm{cm}^{-2}$. The data $(\bullet)$ were obtained in a $\mathrm{MC}$ simulation with the same conditions and an ion diameter of $300 \mathrm{pm}$.

In order to estimate the effective charge on a polyatomic species in the double layer, one must know the potential distribution as a function of distance from the metal/solution interface. In the absence of ionic specific adsorption, the potential in the inner layer is assumed to vary linearly with distance from a value $\phi^{m}$ in the metal to a value $\phi^{d}$ at the oHp. In the diffuse layer, the potential profile is commonly assumed to follow the Gouy-Chapman theory. Accordingly, the potential is given by

$$
\phi=\frac{4 R T}{F} \tanh ^{-1}\left[\tanh \left(\frac{F \phi^{d}}{4 R T} \exp \left(-\kappa x+\kappa x_{d}\right)\right)\right],
$$

where $x_{d}$ is the distance of the oHp from the metal/solution interface, and $\kappa$, the Debye-Huckel reciprocal distance. The resulting potential profile in the diffuse layer is shown in figure 5 for the case of a $1-1$ electrolyte at a concentration of $0.1 \mathrm{M}$, and an electrode charge density of $10 \mu \mathrm{C} \mathrm{cm}^{-2}$. Having made assumptions about 
the position and orientation of the reactant in the double layer, it is now a simple matter to estimate the effective reactant charge using equation (6). Obviously the result depends on the model chosen to describe the potential profile. In this regard it is well known that the Gouy-Chapman theory is defective because it neglects the finite size of the ions in the double layer [11]. Some idea of the effects of including ion size on double layer properties may be obtained from Monte Carlo (MC) data obtained in a primitive level simulation of double layer properties [12]. The chief conclusion of these simulations is that the oHp potential is overestimated by the GC theory. A plot of the potential distribution in the diffuse layer, obtained in a MC simulation for an electrolyte concentration of $0.1 \mathrm{M}$ and an electrode charge density of $10 \mathrm{C} \mathrm{cm}^{-2}$, is also shown in figure 5 . It is clear from a comparison of the GC and $\mathrm{MC}$ results that the functional form of the potential profile in the diffuse layer is similar. It follows that the value of $z_{\text {eff }}$ estimated according to equation (6) does not depend significantly on which potential profile is used.

The effective charge $z_{\text {eff }}$ may also be estimated from kinetic data for the electroreduction of complex ions, such as those considered above, at polarizable electrodes. When the electrode is polarizable, the equilibrium properties of the electrode/solution interface may be obtained in the absence of a reaction. More specifically, the electrode charge density is measured as a function of electrode potential so that the potential drop across the diffuse layer may be estimated.

According to Frumkin's theory [8], the potential dependence of the logarithm of the forward rate constant for the heterogeneous transfer of one electron is given by the equation

$$
\ln k_{f}=\ln k_{0}-z_{\mathrm{eff}} \phi^{d}+\alpha f\left(\phi^{d}-\phi^{m}\right)
$$

where $k_{f}$ is the forward rate constant, $k_{0}$, its value at the point of zero charge (pzc), $\alpha$, the transfer coefficient, and $f=F / R T$. When the kinetic data are sufficiently precise, they may be numerically differentiated [13] to obtain

$$
\frac{R T}{F} \cdot \frac{\mathrm{d} \ln k_{f}}{\mathrm{~d}\left(\phi^{m}-\phi^{d}\right)}=-\alpha+z_{\text {eff }} \frac{\mathrm{d} \phi^{d}}{\mathrm{~d}\left(\phi^{m}-\phi^{d}\right)} .
$$

It follows that a plot of $\left(R T \mathrm{~d} \ln k_{f}\right) /\left(F_{d}\left[\phi^{m}-\phi^{d}\right]\right)$ against should be linear with a slope equal to $z_{\text {eff }}$. This type of analysis was used recently by Hromadova and Fawcett [14] to estimate the effective charge for $\left[\mathrm{Co}\left(\mathrm{NH}_{3}\right)_{5} \mathrm{~F}\right]^{2+}$ reacting in the double layer at single crystal gold electrodes. Otherwise, $z_{\text {eff }}$ is determined by constructing corrected Tafel plots of the kinetic data. In such a plot the logarithm of the forward rate constant is corrected for the variation in reactant concentration in the double layer and the Frumkin equation is written as

$$
\ln k_{f}+z_{\text {eff }} f \phi^{d}=\ln k_{0}+\alpha f\left(\phi^{d}-\phi^{m}\right) .
$$

It follows that a plot of $\ln k_{f}+z_{\text {eff }} f \phi^{d}$ with the correct value of $z_{\text {eff }}$ should be linear in $\phi^{d}-\phi^{m}$ [8]. This is tested further experimentally by obtaining kinetic data at a variety of polarizable metals with different pzc's [14-16], or by varying $\phi^{d}$ over a wide range by varying the electrolyte concentration [10]. All of these methods 
Table 4. Effective charge on the reactant for the reaction $A^{z+}+e^{-} \rightarrow A^{(z-1)+}$.

\begin{tabular}{|l|l|l|l|}
\hline Reaction & Electrode & Electrode charge & $z_{\text {eff }}$ \\
\hline$\left[\mathrm{Co}\left(\mathrm{NH}_{3}\right)_{6}\right]^{3+/ 2+}[14]$ & $\mathrm{Au}$ & positive & $2.0 e_{0}$ \\
{$\left[\mathrm{Fe}\left(\mathrm{H}_{2} \mathrm{O}\right)_{6}\right]^{3+/ 2+}[15]$} & $\mathrm{Au}$ & positive & $2.0 e_{0}$ \\
{$\left[\mathrm{Co}\left(\mathrm{NH}_{3}\right)_{5} \mathrm{~F}\right]^{2+/ 1+}[16]$} & $\mathrm{Au}$ & positive & $1.6 e_{0}$ \\
{$\left[\mathrm{Cr}\left(\mathrm{H}_{2} \mathrm{O}\right)_{6}\right]^{3+/ 2+}[10]$} & $\mathrm{Hg}$ & negative & $2.2 e_{0}$ \\
{$\left[\mathrm{Eu}\left(\mathrm{H}_{2} \mathrm{O}\right)_{8}\right]^{3+/ 2+}[10]$} & $\mathrm{Hg}$ & negative & $1.7 e_{0}$ \\
\hline
\end{tabular}

have been applied with considerable success. Values of $z_{\text {eff }}$ obtained for five different reactions in recent experimental studies are summarized in table 4 . In all cases the value of $z_{\text {eff }}$ is smaller than the nominal charge on the reactant. The anion used in these experiments was always perchlorate. It is well known that this anion is not involved in significant ion pairing in aqueous solutions so that ion pairing cannot be cited as the reason for the lower values of $z_{\text {eff }}$. Furthermore, the results were obtained both under conditions in which the reactant is attracted into and repelled from the double layer. The most convincing explanation of the low values of $z_{\mathrm{eff}}$ is that the reactant is partly in a region of lower electrical potential. In addition, the values of $z_{\text {eff }}$ obtained from the kinetic data were compared with those estimated using a suitable potential profile in the double layer. The two values were always in excellent agreement $[9,10]$.

\section{Discussion}

The importance of knowing the charge distribution in polyatomic ions has been illustrated here using two very different examples, one dealing with ion solvation in solution and the other with double layer effects in electrode kinetics. There are other examples that could be considered. An important problem in electrolyte solutions is ion pairing and its role in determining the activity of electrolytes. Ion pairing is normally discussed with respect to the charge on the ion divided by its radius as a hard sphere. When polyatomic ions are involved in ion pairing the simple picture is not adequate. Other examples are found in the kinetics of homogeneous reactions involving ions. Improved descriptions of electron transfer, proton transfer, and ligand exchange reactions would be possible if the role of charge distribution in polyatomic reactants were considered. In summary, this is an important problem that requires more attention by chemists involved with solution chemistry.

\section{Acknowledgements}

The financial support of the National Science Foundation, Washington (CHE0133758) is gratefully acknowledged. 


\section{References}

1. Guillot B., J. Molec. Liquids, 2002, 101, 219.

2. Bernal J.D., Fowler R.H., J. Chem. Phys., 1933, 1, 515.

3. Krienke H., Schmeer G., Z. Phys. Chem., 2004, 218, 1.

4. Fawcett W.R. Liquids, Solutions and Interfaces, Chap. 3. Oxford University Press, New York, 2004.

5. Tissandier M.D., Cowan K.A., Feng W.Y., Grundlach E., Cohen M.H., Earhart A.D., Cox J.V., Tuttle Jr. T.R., J. Phys. Chem. A, 1998, 102, 7787.

6. Shannon R.D., Prewitt C.T., Acta Crystallogr. B, 1969, 25, 925.

7. Marcus Y. Ion Solvation, Chap. 6. Wiley-Interscience, New York, 1985.

8. Frumkin A.N., Petrii O.A., Nikolaeva-Fedorovich N.V., Electrochim. Acta, 1963, 8, 177.

9. Fawcett W.R., Hromadova M., Tsirlina G.A., Nazmutdinov R.R., J. Electroanal. Chem., 2001, 498, 93.

10. Rusanova M.Yu., Tsirlina G.A., Nazmutdinov R.R., Fawcett W.R., J. Phys. Chem. B, 2005, 109, 1348.

11. Fawcett W.R., Henderson D.J., J. Phys. Chem. B, 2000, 104, 6837.

12. Boda D., Fawcett W.R., Henderson D., Sokolowski S., J. Chem. Phys., 2002, 116, 7170 .

13. Fawcett W.R., Kent J.E., Kuo Lee Y.C., J. Electroanal. Chem., 1969, $20,357$.

14. Hromadova M., Fawcett W.R., J. Phys. Chem. B, 2004, 108, 3277.

15. Hromadova M., Fawcett W.R., J. Phys. Chem. A, 2000, 104, 4356.

16. Hromadova M., Fawcett W.R., J. Phys. Chem. A, 2001, 105, 104. 


\section{Ефекти розподілу заряду у хімії розчинів} поліатомних іонів

\section{У.Р.Фоцетт}

Факультет хімії, університет Каліфорнії, Девіс, США

Отримано 15 листопада 2004 р.

Розподіл заряду в поліатомних іонах, отриманий на основі ab initio квантово хімічних розрахунків, використовується для детального опису хімії їхнього розчину. Два приклади розглядаються в даній статті. Солватація малих поліатомних іонів, таких як нітрати та перхлорати, розглядається відповідно до MSA моделі іонної солватації. Інший приклад включає розгляд ефектів подвійного шару для комплексів перехідних металів в умовах електронного обміну у подвійному шарі.

Ключові слова: солватація, поліатомні іони

PACS: 82.60.Lf 